\title{
Exposição dos agricultores do Posto Administrativo de Chaimite aos pesticidas agrícolas
}

\author{
Exposure to agricultural pesticides by farmers of Chaimite administrative post
}

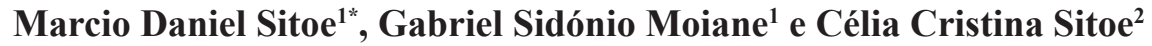

Submissão: 18/02/2016 / Aceite: 13/05/2017

\section{RESUMO}

O uso dos pesticidas agrícolas, é de fundamental importância na redução das perdas causadas por pragas e doenças, não obstante os riscos à saúde humana e ao ambiente. Este trabalho, objetivou analisar a exposição dos agricultores do Posto Administrativo (PA) de Chaimite, Distrito de Chibuto, Província de Gaza, aos pesticidas agrícolas. Para a sua materialização, foi usado um questionário misto, dirigido a 40 agricultores do PA de Chaimite. Constatou-se que os pesticidas mais utilizados são o Mancozebe, a Cipermetrina e Metamidofos, que de forma geral, variam de pouco tóxicos (Mancozebe) aos altamente tóxicos (Metamidofos). Os aplicadores não estão suficientemente treinados para o trabalho seguro e eficiente com os pesticidas, além de não possuírem o EPI completo (Equipamento de Proteção Individual), o que os torna altamente expostos, e passíveis à intoxicações, que podem levar a perda de vidas. Deste modo, tornam-se necessárias ações que viabilizem o acesso e uso do EPI pelos produtores do setor familiar, e capacitações e/ou treinamento em Tecnologias de aplicação de pesticidas.

PALAVRAS-CHAVE: intoxicação, risco à saúde humana, segurança dos trabalhadores rurais.

\footnotetext{
ABSTRACT

The use of agricultural pesticides, has fundamental importance in reducing losses caused by pests and diseases, despite the risks to human health and the environment. The aim of this study was to analyze the agricultural pesticides exposure of farmers from Chaimite Administrative Post (AP), in Chibuto District, Gaza Province. A mixed questionnaire involving 40 Chaimite AP farmers were used. It

${ }^{1}$ Universidade Eduardo Mondlane, Chibuto, Moçambique.

${ }^{2}$ Escola Secundária de Chokwè, Chokwè, Moçambique.

*Autor para corespondência <marcio.sitoe@uem.mz>
}

was found that the most commonly used pesticides are Mancozeb, Cypermethrin and Methamidophos, which generally range from low toxic (Mancozeb) to very toxic (Methamidophos). The applicators are not sufficiently trained for safe and efficient work with pesticides, apart from not having a full KIT of Personal Protective Equipment (PPE), which makes them highly exposed and susceptible to poisoning, which can lead to loss of life. Therefore, it is necessary to make the access and use of PPE by family sector producers and training in pesticide application Technologies viable.

KEYWORDS: intoxication, risk to human health, rural workers safety.

\section{INTRODUÇÃO}

Os pesticidas são substâncias utilizadas para o controle de pragas agrícolas e vetores de doenças em saúde pública (RAGHAVENDRA et al. 2011, YADOULETON et al. 2011, RANSON et al. 2011, ISMAN et al. 2011, HILLOCKS 2012, PINTO 2015).

$\mathrm{O}$ seu uso na agricultura, contribui para evitar perdas decorrentes da ação nefasta das pragas, permitindo o aproveitamento máximo dos rendimentos obtidos durante a produção. Entretanto, o uso inadequado, é responsável pela intoxicação do homem e de animais domésticos, poluição ambiental e redução das populações de inimigos naturais das pragas no mundo (GEIGER et al. 2010, MEISSLE et al. 2010, POTTS et al. 2010, DYK \& PLETSCHKE 2011, PINTO 2015).

O seu potencial no controle de pragas agrícolas, foi comprovado em vários estudos (REDOAN et al. 2010, BRUSTOLIN et al. 2011, GUEDES et al. 2012, VIEIRA et al. 2012). Foi igualmente demonstrada a relação entre a exposição aos pesticidas e a ocorrência 
de doenças como a Leucemia em crianças (TURNER et al. 2011, FERREIRA et al. 2012), a doença de Parkinson (PEREIRA \& GARRETT 2010, BARTH \& BIAZON 2010), as lesões no sistema auditivo (KORBES et al. 2010, KÓS et al. 2013), efeitos deletérios sobre parâmetros seminais (CASTRO et al. 2014, MINGUEZ-ALÁRCON 2014) e ao fraco desenvolvimento cognitivo e/ou neuropatia (SOUZA et al. 2011, CAMPOS et al. 2015).

Em Moçambique, de forma geral, os pesticidas agrícolas são muito pouco utilizados, principalmente, pelos agricultores do setor familiar com fraco acesso ao mercado, escassez de recursos e cuja produção se destina à subsistência (JOSSEFA et al. 2014, SITOE 2014, GUANZIROLI \& GUANZIROLI 2015), não obstante, nos últimos 10 anos, a importação destes produtos ter aumentado em cerca de 500\%, aumentando o risco de ocorrência de problemas decorrentes do seu uso.

Nos sistemas de agricultura familiar em Moçambique, a preparação e aplicação dos pesticidas, é na sua maioria feita por membros da família, havendo maior participação dos homens relativamente às mulheres e crianças. Apesar da participação das crianças ser pouco comum, é notável na aplicação, em comparação com a preparação dos pesticidas.

De forma geral, os produtos mais utilizados, apresentam toxicidade média a alta, alguns dos quais, já banidos em alguns países, como é o caso do Metamidofos no Brasil (ANVISA 2011).

Estes produtos, preparados e aplicados por produtores pouco treinados e sem acesso aos equipamentos de proteção individual (EPI), representam forte ameaça à saúde. O risco estende-se a todos os intervenientes nas zonas de produção e de consumo, quer pela exposição indireta aos pesticidas no meio rural, como pelo consumo de produtos alimentares contendo resíduos destes produtos.

Deste modo, com o presente trabalho, pretendese analisar a exposição dos agricultores aos pesticidas utilizados no Posto Administrativo de Chaimite, Distrito de Chibuto, Província de Gaza, Moçambique.

\section{MATERIAL E MÉTODOS}

Foi realizado um estudo de natureza quantitativa e qualitativa, envolvendo agricultores do Posto Administrativo (PA) de Chaimite, no Distrito de Chibuto, Província de Gaza, Moçambique.

Procurou-se estudar o nível de acesso e uso dos pesticidas, equipamentos de protecção individual, e o conhecimento das Tecnologias de Aplicação de Pesticidas pelos agricultores locais.

A coleta da informação foi realizada em duas fases:

A primeira, consistiu em um estudo diagnóstico, com recurso às entrevistas semi-estruturadas, envolvendo as autoridades locais, extensionistas, responsáveis pelas associações de produtores e responsáveis pelos serviços públicos de agricultura. Nesta fase, o objetivo foi o de coletar informação de suporte para a implementação do inquérito aos produtores.

A segunda, consistiu no inquérito aplicado aos agricultores do PA de Chaimite, na sua maioria proprietários das unidades produtivas, nas quais cultivam milho, feijões e hortícolas.

A amostra usada, foi de 40 agricultores, dos quais, 18 homens e 22 mulheres.

Para a escolha dos inquiridos, as unidades produtivas foram enumeradas e através de uma lista de números aleatórios, foram selecionadas 40 unidades, nas quais, os proprietários ou seus representantes foram inquiridos.

Todos os agricultores de Chaimite tiveram a mesma oportunidade de serem escolhidos e em situações de ausência dos entrevistados na propriedade e/ou unidade de produção, os inquéritos eram administrados em dias subsequentes.

Os dados foram analisados no programa SPSS versão 17 e os resultados apresentados em percentagens.

\section{RESULTADOS E DISCUSSÃO}

\section{Aquisição e aplicação de pesticidas}

Os agricultores do setor familiar no PA de Chaimite, apresentam dificuldades financeiras para a aquisição dos pesticidas necessários e suficientes para o controle de pragas e doenças que ciclicamente atacam culturas agrícolas, contribuindo significativamente para a ocorrência de focos de insegurança alimentar. Apesar disso, 85\% dos inquiridos afirmam aplicar pesticidas em todas as campanhas agrícolas, enquanto os restantes $15 \%$ fazem-no em algumas campanhas. Os principais pesticidas utilizados são o Mancozebe, Cipermetrina e Metamidofos, aplicados por cerca de 90,78 e $53 \%$ dos agricultores, respectivamente, (Tabela 1).

$\mathrm{O}$ fato dos agricultores de Chaimite aplicarem 
pesticidas em praticamente todas as campanhas agrícolas, reflete de certa forma, a convicção que se tem, de que o controle químico é a tática mais eficiente no controlo de pragas naquele PA, fato que contribui para o fraco uso do Manejo Integrado de Pragas, pelos agricultores locais. Entretanto, a falta de recursos financeiros para a aquisição de pesticidas, limita de certa forma a aplicação destes produtos em todas as áreas produtivas, por todos os produtores e em quantidades superiores as utilizadas atualmente.

Ademais, as áreas dos agricultores sem recursos suficientes para a aquisição de pesticidas, servem de abrigo para as pragas e seus inimigos naturais, permitindo a ocorrência do controle biológico e consequentemente, a redução da velocidade da queda das populações de inimigos naturais e da biodiversidade em geral, evitando também a rápida seleção de pragas resistentes aos pesticidas utilizados (SANTOS et al. 2011, GODOY et al. 2010).

A crescente preocupação com os efeitos nefastos causados pelos pesticidas aplicados em Chaimite sobre as populações de inimigos naturais de pragas, pode ser suportada por vários estudos que mostram tais efeitos sobre insetos benéficos, incluindo polinizadores (CASTILHOS et al. 2011, SCARPELLINI \& ANDRADE 2011, LAURINO et al. 2011, MENEZES et al. 2012, BLACQUIÈRE et al. 2012, COSTA et al. 2014).

Dos principais pesticidas aplicados pelos agricultores de Chaimite, o Metamidofos foi banido no Brasil (ANVISA 2011). Apesar da comprovada eficiência no controle de várias espécies de pragas, tanto este pesticida de largo espectro de ação, como a Cipermetrina, o Mancozebe e outros, estão relacionados a riscos à saúde humana (DIAZ 2012, PINTO-ZAVALHOS \& ZARBIN 2013, OLIVEIRA \& LUCCHESE 2013), à toxicidade à abelhas (POTTS et al. 2010, JOHNSON et al. 2010, HENRY et al. 2012), aos peixes (SARAVANAN et al. 2011, MURTHY et al. 2013, AMÉRICO et al. 2015), à animais domésticos (BERNY et al. 2010, DELLARCO et al. 2010, RUIZSUÁREZ et al. 2015), à contaminação de recursos hídricos (ANDRADE et al. 2011, DELLAMATRICE \& MONTEIRO 2014, POSSAVATZ et al. 2014), entre outros.

Tabela 1. Assistência técnica, aquisição e uso de pesticidas agrícolas pelos agricultores do Posto Administrativo de Chaimite.

Table 1. Technical assistance, acquisition and use of agricultural pesticides by Chaimite Administrative Post farmers.

\begin{tabular}{lcc}
\hline \multicolumn{1}{c}{ Questões colocadas } & Opções de resposta & Percentagem (\%) de agricultores \\
\hline Uso de pesticidas em & Sempre & 85 \\
campanhas agrícolas & Algumas vezes & 15 \\
Disponibilidade de receita & Sempre & 15 \\
agronômica no ato da & Algumas vezes & 7,5 \\
aquisição dos pesticidas & Nunca & 77,5 \\
\cline { 2 - 3 } & Técnicos do SDAE ${ }^{1}$ & 45 \\
& Amigos ou familiares & 10 \\
Assistência aos agricultores & Propaganda & 2,5 \\
na aquisição e aplicação de & Revendedores de pesticidas & 20 \\
pesticidas & Proprietário das áreas de & 20 \\
& produção & 2,5 \\
Verificação da validade dos & Outros agricultores & 71,4 \\
pesticidas antes da compra & Sempre & 28,6 \\
\hline
\end{tabular}

${ }^{1}$ Serviços Distritais de Actividades Económicas 
Estes agricultores, recebem orientações sobre os pesticidas a adquirirem e a aplicarem. As orientações são oferecidas por diferentes intervenientes da sociedade, sendo que os Técnicos dos Serviços Distritais da Agricultura contribuem em $45 \%$, os revendedores de pesticidas em $20 \%$, os proprietários das áreas de produção em $20 \%$, os familiares e amigos em 10\%, outros agricultores em $2,5 \%$ e as propagandas em $2,5 \%$ (Tabela 1 ).

Há que salientar que, apesar de não constituir prática habitual em Moçambique, constatou-se que para a aquisição dos pesticidas, $22,5 \%$ dos entrevistados, utilizam receita agronômica prescrita pelos extensionistas ligados aos Serviços Distritais da Agricultura. Outrossim, metade dos inquiridos referem que avaliam o estado de conservação das embalagens dos pesticidas antes de efetuar a compra, e $71,4 \%$ verificam sempre a validade dos produtos antes de comprá-los (Tabela 1).

A qualidade dos Serviços de Extensão prestados às comunidades, é determinante na orientação dos agricultores do setor familiar em relação a aquisição e uso dos pesticidas.

Em Moçambique, por um lado, o número, a formação e as condições de trabalho dos extensionistas agrários, estão longe do ideal, o que impõe enormes desafios ao País, por outro lado, o fato de a recomendação técnica incluir o uso de pesticidas com alta toxicidade, traz o desafio da atualização regular dos pesticidas autorizados e recomendados para uso agrícola, em consonância com a evolução dos estudos de toxicidade e ecotoxicidade destes produtos no mundo, mesmo que isso implique a restrição parcial do uso de pesticidas com alta toxicidade, pelos agricultores do setor familiar sem acesso a Equipamentos de Proteção Individual e treinamento adequados.

\section{Pulverizadores utilizados}

À imagem da realidade nacional, maior parte dos agricultores de Chaimite, utilizam Pulverizadores costais (90\%). Os proprietários destes equipamentos, afirmam que realizam a manutenção de forma regular, apesar de terem sido observados muitos pulverizadores em mau estado de conservação (Tabela 2).

Agricultores bem treinados e protegidos com Equipamentos de Proteção Individual (EPI) garantem o uso eficiente e seguro dos pesticidas.

Deste estudo, constatou-se que pouco mais de $20 \%$ dos inquiridos não conhece as técnicas de aplicação dos pesticidas, o que mostra o fraco treinamento dos agricultores. Todavia, a eficiência das pulverizações, depende em parte, do nível de treinamento dos aplicadores de pesticidas (CASALI et al. 2015).

Verificou-se ainda que, sempre que se deparam com pontas de pulverização entupidas, os agricultores que afirmam possuir conhecimento das técnicas de aplicação, desentopem-nas utilizando agulhas (60\%), ramos de árvores $(12,5 \%)$, enquanto $5 \%$ preferem sacudir. Aqueles sem conhecimento das técnicas de aplicação, utilizam água $(17,5 \%)$ e ramos de árvores (5\%) (Tabela 2).

\section{Destino das embalagens vazias dos pesticidas}

As embalagens vazias dos pesticidas, são jogadas no meio ambiente (17,5\%), enterradas $(22,5 \%)$ ou queimadas (60\%) (Tabela 3). Porém, o destino dado, não é o mais adequado por contribuir para a poluição ambiental, do solo, das águas e do ar que respiramos. Esta forma de agir, é consequência da falta de uma política pública de logística reversa das embalagens vazias de pesticidas, que inclua a obrigatoriedade da devolução das embalagens vazias ao revendedor dos pesticidas, para serem tratadas de forma adequada em entidades concebidas para o efeito (MOTTA NOGUEIRA \& DANTAS 2013, SOUZA 2014, MARQUES \& VIEIRA 2015).

\section{Uso de Equipamentos de Proteção Individual}

Todos os agricultores inquiridos são unânimes quanto à necessidade do uso dos EPI durante o manejo, preparação e aplicação dos pesticidas, de forma a evitar-se o contato direto com os produtos. Entretanto, nenhum inquirido possui o EPI completo. Assim, na prática, os $87,5 \%$ que afirmam ter EPI, possuem apenas alguns elementos do lote, sendo as botas de borracha e luvas, os mais comuns (Tabela 3).

A importância dos EPI na proteção dos aplicadores de pesticidas, é consensual entre cientistas (MARTINS et al. 2012, FEOLA et al. 2012, RIBEIRO et al. 2014), porém, a falta de recursos, limita o uso generalizado pelos agricultores de Chaimite, expondo-os aos riscos dos pesticidas.

Os inquiridos estão cientes dos riscos associados aos pesticidas, todavia, apenas $22,5 \%$ afirmam ter presenciado casos de intoxicação de pessoas e/ou animais. 
Tabela 2. Tipo, estado de conservação e manutenção dos pulverizadores utilizados pelos agricultores do Posto Administrativo de Chaimite.

Table 2. Type, condition and maintenance of sprays used by Chaimite Administrative Post farmers.

\begin{tabular}{lcc}
\hline \multicolumn{1}{c}{ Questões colocadas } & Opções de resposta & $\begin{array}{c}\text { Percentagem (\%) de } \\
\text { agricultores }\end{array}$ \\
\hline Pulverizadores utilizados & Costais & 90 \\
pelos agricultores & Acoplados ao trator e & 10 \\
cstado de conservação dos & Muito bom & 7,5 \\
pulverizadores & Bom & 87,5 \\
& Ruim & 5 \\
\cline { 2 - 3 } Formas de limpeza dos bicos & Com ramos de árvores & 17,5 \\
dos pulverizadores & Com água & 17,5 \\
& Agulhas & 60 \\
Conhecimento das técnicas & Sacudindo & 5 \\
de preparação e aplicação de & Conhecem & \\
pesticidas & & 22,5 \\
\hline
\end{tabular}

Tabela 3. Destino das embalagens vazias de pesticidas e uso dos Equipamentos de Proteção Individual. Table 3. Destination of empty pesticide containers and use of Personal Protective Equipment.

\begin{tabular}{lcc}
\hline \multicolumn{1}{c}{ Questões colocadas } & Opções de resposta & Percentagem (\%) de agricultores \\
\hline & Jogam no meio ambiente & 17,5 \\
Destino das embalagens & Enterram & 22,5 \\
vazias de pesticidas & Queimam & 60 \\
Agricultores com pelo menos & Possuem & 87,5 \\
\cline { 2 - 3 } uma peça do Equipamento de & Não possuem & 12,5 \\
Proteção Individual & & \\
\hline
\end{tabular}




\section{CONCLUSÕES}

Os agricultores do Posto Administrativo de Chaimite, não possuem o EPI completo nem treinamento em tecnologias de aplicação de pesticidas.

Utilizam alguns pesticidas altamente tóxicos, $o$ que os coloca em elevada exposição a estes produtos, com probabilidade de ocorrência de intoxicações que podem levar a perda de vidas humanas.

\section{REFERÊNCIAS}

AMÉRICO JHP et al. 2015. Uso de agrotóxicos e os impactos nos ecossistemas aquáticos. Revista Científica ANAP Brasil 8: 101-115.

ANDRADEAS et al.2011. Análise de risco de contaminação de águas superficiais e subterrâneas por pesticidas em Municípios do Alto Paranaíba - MG. Química Nova 34: 1129-1135.

ANVISA - Agência Nacional de Vigilância Sanitária. 2011. Resolução - RDC No 1, de 14 de Janeiro de 2011. Regulamento técnico para o ingrediente ativo Metamidofós em decorrência da reavaliação toxicológica. Diário Oficial da União, Brasília, 17 jan.

BARTH VG \& BIAZON ACB. 2010. Complicações decorrentes da intoxicação por organofosforados. SaBios: Revista de Saúde e Biologia 5: 27-33.

BERNY P et al. 2010. Animal poisoning in Europe. Part 2: Companion animals. The Veterinary Journal 183: 255-259. BLACQUIĖRE $\mathrm{T}$ et al. 2012. Neonicotinoids in bees: a review on concentrations, side-effects and risk assessment. Ecotoxicology 21: 973-992.

BRUSTOLIN $\mathrm{C}$ et al. 2011. Inseticidas em pré e pós emergência do milho (Zea mays L.), associados ao tratamento de sementes, sobre Dichelops melacanthus (Dallas) (Hemiptera: Pentatomidae). Revista Brasileira de Milho e Sorgo 10: 215-223.

CAMPOS E et al. 2015. Exposição a pesticidas organoclorados e desenvolvimento cognitivo em crianças e adolescentes residentes em uma área contaminada no Brasil. Revista Brasileira de Saúde Materno Infantil 15:105-120.

CASALI AL et al. 2015. Nível de capacitação e informação dos operadores de máquinas para a aplicação de agrotóxicos. Ciência Rural 45: 425-431.

CASTILHOS RV et al. 2011. Seletividade de agrotóxicos utilizados em pomares de pêssego a adultos do predador Chrysoperla externa (Hagen, 1861) (Neuroptera: Chrysopidae). Revista Brasileira de Fruticultura 33: 73-80. CASTRO HFB et al. 2014. Influência dos agrotóxicos na qualidade seminal: Uma revisão da literatura. Revista Unimontes Científica 16:72-78.

COSTA EM et al. 2014. Toxicity of insecticides used in the Brazilian melon crop to the honey bee Apis mellifera under laboratory conditions. Apidologie 45: 34-44.
DELLAMATRICE PM \& MONTEIRO RTR. 2014. Principais aspectos da poluição de rios brasileiros por pesticidas. Revista Brasileira de Engenharia Agrícola e Ambiental 18: 1296-1301.

DELLARCO VL et al. 2010. A retrospective analysis of toxicity studies in dogs and impact on the chronic reference dose for conventional pesticide chemicals. Critical Reviews in Toxicology 40: 16-23.

DIAZ MB. 2012. Risco Ecotoxicológico do Metamidofós. Revista Intertox de Toxicologia, Risco Ambiental e Sociedade 5: 62-76.

DYK JSV \& PLETSCHKE B. 2011. Review on the use of enzymes for the detection of organochlorine, organophosphate and carbamate pesticides in the environment. Chemosphere 82: 291-307.

FEOLA G. et al. 2012. Exploring behavioural change through na agent-oriented system Dynamics model: The use of personal protective equipment among pesticide applicators in Colombia. System Dynamics Review 28: 6993.

FERREIRA JD et al. 2012. Exposições ambientais e leucemias na infância no Brasil: uma análise exploratória de sua associação. Revista Brasileira de Estudos de População 29: 477-492.

GEIGER $F$ et al. 2010. Persistent negative effects of pesticides on biodiversity and biological control potential on European Farmland. Basic and Applied Ecology 11: $97-$ 105.

GODOY KB et al. 2010. Parasitismo e sítios de diapausa de adultos do percevejo marron, Euschistus heros na região de Grande Dourados. Ciência Rural 40: 1199-1202.

GUANZIROLI CE \& GUANZIROLI T. 2015. Modernização da agricultura em Moçambique: determinantes da renda agrícola. Revista de Economia e Sociologia Rural 53: 115-128.

GUEDES JVC et al. 2012. Sistemas de aplicação e inseticidas no controle de Anticarsia gemmatalis na soja. Revista Brasileira de Engenharia Agrícola e Ambiental 16: 910-914.

HENRY M et al. 2012. A common pesticide decreases foraging success and survival in honey bees. Science 336: 348-350.

HILLOCKS RJ. 2012. Farming with fewer pesticides: EU pesticide review and resulting challenges for UK agriculture. Crop Protection 31: 85-93.

ISMAN MB et al. 2011. Commercial opportunities for pesticides based on plant essential oils in agriculture, industry and consumer products. Phytochemistry Reviews 10: 197-204.

JOHNSON RM et al. 2010. Pesticides and honey bee toxicity-USA. Apidologie 41: 312-331.

JOSSEFA M et al. 2014. Desenvolvimento comunitário e ambiente: Caso das associações apoiadas pela Associação Mozal para o Desenvolvimento da Comunidade (Maputo, Moçambique). CAPTAR: Ciência e Ambiente Para Todos 
5: 50-62.

KORBES D et al. 2010. Alterações no sistema vestibulococlear decorrentes da exposição ao agrotóxico: revisão da literatura. Revista da Sociedade Brasileira de Fonoaudiologia 15: 146-152.

KÓS MI et al. 2013. Efeitos da exposição a agrotóxicos sobre o sistema auditivo periférico e central: Uma revisão sistemática. Cadernos de Saúde Pública 28: 1491-1506.

LAURINO D et al. 2011. Toxicity of neonicotinoid insecticides to honey bees: laboratory tests. Bulletin of Insectology 64:107-113.

MARQUES MD \& VIEIRA SC. 2015. Produtores rurais em localidades do Interior Paulista como Tupã e a logística reversa de devolução das embalagens vazias de agrotóxicos. Revista Científica ANAP Brasil 8: 30-46.

MARTINS MKS et al. 2012. Exposição ocupacional aos agrotóxicos: Um estudo transversal. Revista Intertox de Toxicologia, Risco Ambiental e Sociedade 5: 6-27.

MEISSLE $M$ et al. 2010. Pests, pesticide use and alternative options in European maize production: current status and future prospects. Journal of Applied Entomology 134: 357375.

MENEZES CWG et al. 2012. Seletividade de atrazine e nicosulfuron a Podisus nigrispinus (Heteroptera: Pentatomidae). Planta daninha 30: 327-334.

MINGUEZ-ALÁRCON L et al. 2014. Calidad seminal y toxicidad de metales pesados y plaguicidas. Revista Salud Ambiental 14: 8-19.

MOTTA NOGUEIRA V \& DANTAS R. 2013. Gestão ambiental de embalagens vazias de agrotóxicos. Tema Revista Eletrônica de Ciências 14: 22-34.

MURTHY KS et al. 2013. A review on toxicity of pesticides in Fish. International Journal of Open Scientific Research 1: 15-36.

OLIVEIRA KM \& LUCCHESE G. 2013. Controle sanitário de agrotóxicos no Brasil: o caso do metamidofós. Revista Tempus Actas de Saúde Coletiva 7: 211-224.

PEREIRA D \& GARRETT C. 2010. Factores de risco da doença de Parkinson: Um estudo epidemiológico. Acta Médica Portuguesa 23: 15-24.

PINTO GMF. 2015. Os pesticidas, seus riscos e movimento no meio ambiente. Revista Eletrônica FACP 8: 1-12.

PINTO-ZEVALLOS DM \& ZARBIN PHG. 2013. A química na agricultura: perspectivas para o desenvolvimento de tecnologias sustentáveis. Química Nova 36: 1509-1513. POSSAVATZ J et al. 2014. Resíduos de pesticidas em sedimento de fundo de rio na Bacia Hidrográfica do Rio Cuiabá, Mato Grosso, Brasil. Revista Ambiente \& Água 9: 88-96.

POTTS SG et al. 2010. Global pollinator declines: trends, impacts and drivers. Trends in Ecology and Evolution 25: 345-353.

RAGHAVENDRA K et al. 2011. Malaria vector control: From past to future. Parasitology Research 108: 757-779.

RANSON H et al. 2011. Pyrethroid resistance in African anopheline mosquitoes: What are the implications for malaria control? Trends in Parasitology 27: 91-98.

REDOAN AC et al. 2010. Efeitos de inseticidas usados na cultura do milho (Zea mays L.) sobre ninfas e adultos de Doru leteipes (Scudder) (Dermaptera: Forficulidae) em semicampo. Revista Brasileira de Milho e Sorgo 9: 223235.

RIBEIRO E et al. 2014. Segurança e saúde do aplicador de agrotóxicos: Agricultores do Município de São Joaquim do Monte - PE. Revista de Geografia 31: 39-57.

RUIZ-SUÁREZ N et al. 2015. Continued implication of the banned pesticides carbofuran and aldicarb in the poisoning of domestic and wild animals of the Canary Islands (Spain). Science of the Total Environment 505: 1093-1099.

SANTOS VC et al. 2011. Insecticide resistance in populations of the diamondback moth, Plutella xylostella (L.) (Lepidoptera: Plutellidae), from the state of Pernambuco, Brazil. Neotropical Entomology 40: 264-270. SARAVANAN $M$ et al. 2011. Haematological and biochemical responses of freshwater teleost fish Cyprinus carpio (Actinopterygii: Cypriniformes) during acute and chronic sublethal exposure to lindane. Pesticide Biochemistry and Physiology 100: 206-211.

SCARPELLINI JR \& ANDRADE DJ. 2011. Efeito de inseticidas sobre a joaninha Cycloneda sanguinea L. (Coleoptera, Coccinellidae) e sobre o pulgão Aphis gossypii Glover (Hemiptera, Aphididae) em algodoeiro. Arquivos do Instituto Biológico de São Paulo 78: 393-399.

SITOE TA. 2014. Os desafios da investigação agrária em Moçambique. Desenvolvimento em Questão 12: 81-104.

SOUZA A et al. 2011. Avaliação do impacto da exposição a agrotóxicos sobre a saúde da população rural. Vale do Taquari (RS, Brasil). Ciência \& Saúde Coletiva 16: 35163528.

SOUZA VN \& GLEBER L. 2014. Análise de cenário envolvendo embalagens vazias de agrotóxicos originadas da cultura da Macieira. Pesticidas: Revista de Ecotoxicologia e Meio Ambiente 23: 75-82.

TURNER MC et al. 2011. Residential pesticides and childhood leukemia: a systematic review and metaanalysis. Ciência \& Saúde Coletiva 16: 1915-1931.

VIEIRA SS et al. 2012. Efeitos dos inseticidas utilizados no controle de Bemisia tabaci (Gennadius) biótipo B e sua seletividade aos inimigos naturais na cultura da soja. Semina: Ciências Agrárias 33: 1809-1818.

YADOULETON A et al. 2011. Cotton pest management practices and the selection of pyrethroid resistance in Anopheles gambiae population in Northern Benin. Parasites \& Vectors 4:60. 